\title{
Photometric and spectroscopic analysis of the stellar association AB Doradus
}

\author{
Orlando J. Katime-Santrich ${ }^{1,2}$, Bruno V. Castilho ${ }^{2}$, Carlos A. O. \\ Torres $^{2}$, and Germano R. Quast ${ }^{2}$ \\ ${ }^{1}$ Universidade Federal de Itajubá UNIFEI \\ Itajubá, Minas gerais, Brazil \\ email: osantrich@lna.br \\ ${ }^{2}$ Laboratorio Nacional de Astrofísica LNA \\ Itajubá, Minas gerais, Brazil
}

\begin{abstract}
We present the stellar parameters and lithium abundance for 23 stars of the young stellar association AB Doradus, determined by photometry and spectroscopy. The photometric data was obtained at OPD/LNA and/or from the literature and the spectroscopic data was obtained at La silla/ESO and at OPD/LNA. The parameters were determined using photometric calibrations, line ratios, curves of growth and spectral synthesis. Our results confirm that the selected stars are probably association members, showing an uniform metallicity and lithium depletion consistent with 50 Myears
\end{abstract}

Keywords. Astrochemistry, stars: abundances, pre-main sequence, atmospheres

\section{Datas and methodology}

The young stellar associations are an important laboratories to understand stellar formation and evolution (e.g. Torres, C. A. O. et al. 2006a, Torres, C. A. O. et al. 2006b, and Zuckerman, B. et al. 2004). da Silva, L. et al. (2009) shows that AB Doradus has 92 known members and has an age of 50 Myears. In this work we perform detailed analysis of 23 member stars based on photometric and spectroscopic data. The photometry was obtained mostly at the Pico dos Dias Observatory (OPD/LNA) in Brazil, with the high velocity photometer FOTRAP installed at the $0.6 \mathrm{~m}$ Zeiss telescope. Additional data was retrieved from Simbad database, Tycho, and Hipparcus catalogs. The spectra were obtained using the FEROS spectrograph at the La silla/ESO $2.2 \mathrm{~m}$ and $1.52 \mathrm{~m}$ telescopes and with the Coude spectrograph at the OPD/LNA $1.6 \mathrm{~m}$ telescope. We performed an initial $T_{\text {eff }}$ estimation using photometric calibrations (Alonso, A. et al. 1996 and Houdashelt, M. L. et al. 2000) and spectral line ratios calibrations of Padgett, D. L. (1996), the $T_{\text {eff }}$ was refined by means of curves of growth using the code Renoir by Monique Spite. The surface gravities were calculated preliminarily by adjusting the ZAMS vs $\log (g)$ and refined by the curve of growth, microturbulence velocities were also obtained from the curves of growth. The preliminary metallicities were obtained through Alonso's polynomial calibration and the final values were calculated using curves of growth of FeI and FeII, and finally, the lithium abundances were calculated through spectral synthesis using the code Spectrum (Gray, R. O. \& Corbally, C. J. 1994).

\section{Results and conclusions}

The determined stellar parameters are in the following ranges of $T_{\text {eff }}=[4486-6031] \mathrm{K}$, $\log (g)=[3.9-4.6],[\mathrm{Fe} / \mathrm{H}]=[0.0 \pm 0.2], V_{m t}=[1.0-2.5] \mathrm{Km} / \mathrm{s}$ and $N(L i)=[0.6-$ 2.9]dex. Our results confirm that the stars identified by Torres, C. A. O. et al. (2006a) 

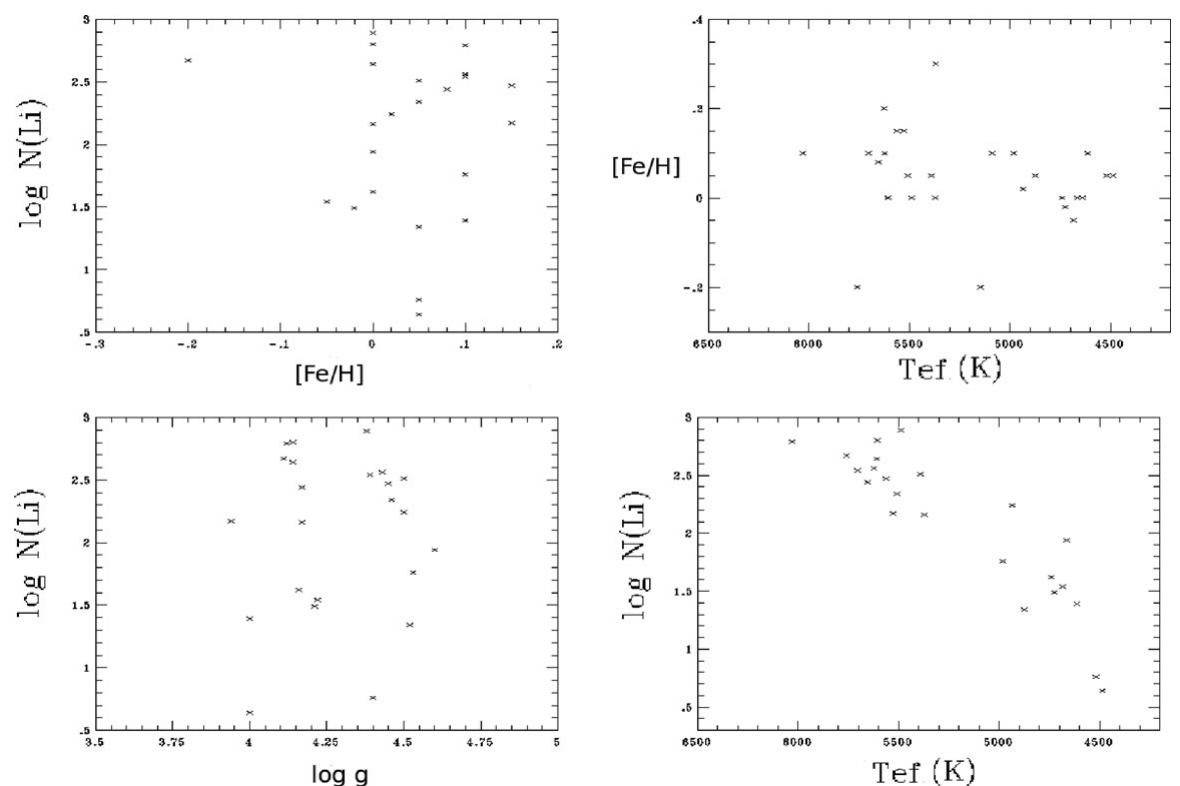

Figure 1. No correlation was found between $N(\mathrm{Li})$ and $[\mathrm{Fe} / \mathrm{H}],[\mathrm{Fe} / \mathrm{H}]$ and $T_{\text {eff }}, N(\mathrm{Li})$ and $\log (g)$ showing that few or no systematic trend was induced by the calculations.

and da Silva, L. et al. (2009), as members of the association, are indeed young stars, having parameters and abundances compatible with the membership in $A B$ Doradus and therefore the association stars can be studied like a whole. The results also seem to confirm some of the hypothesis raised by Travaglio, C.; et al. (2001) concerning the behavior of lithium abundance in the pre-main sequence and main sequence evolution.

\section{References}

Alonso, A., Arribas, S., \& Martinez-Roger, C. 1996, A\&GA 313, 873

da Silva, L., Torres, C. A. O., de la Reza, R., Quast, G. R., Melo, C. H., \& Sterzik, 2009, A\&A accepted

Gray, R. O. \& Corbally, C. J. 1994, AJ 107, 742

Houdashelt, M. L., Bell, R. A., \& Sweigart, A. V. 2000, AJ 119, 1448

Padgett, D. L. 1996, ApJ 451, 1053

Torres, C. A. O., Quast, G. R., da Silva, L., de La Reza, R., Melo, C. H. F., \& Sterzik, M. 2006, $A \mathscr{E} A 460,695$

Torres, C. A. O., Quast, G. R., Melo, C. H., \& Sterzik, 2006, Handbook of Star Forming Regions, Volume II: The Southern Sky ASP Monograph Publications v5, p757 Edited by Bo Reipurth

Travaglio, C., Randich, S., Galli, D., Lattanzio, J., Elliott, L. M., Forestini, M., \& Ferrini, F. 2001 2001, ApJ 559, 909

Zuckerman, B., Song, I., \& Bessell, M. S. The AB Doradus Moving Group 2004, ApJ 613 